\title{
ESTUDO DA LIBERAÇÃO RENAL DE GLICOSE EM COELHOS SUBMETIDOS A HEPATECTOMIA TOTAL FUNCIONAL E INFUSÃO DE NORADRENALINA
}

\author{
Antonio Roberto FRANCHI-TEIXEIRA*, Gracinda L. JORGE**, \\ Luís Sérgio LEONARDI*** e Mário José Abdalla SAAD****
}

RESUMO - Objetivo - Estudar as prováveis fontes endógenas de glicose na ausência do fígado (equivalente à fase anepática do transplante de figado). Material e Métodos - Criou-se um modelo experimental de hepatectomia total funcional em coelhos anestesiados. A aorta e a veia renal direita foram cateterizadas para colheita de glicemias seriadas. Os animais foram divididos em dois grupos: grupo 1, com 5 animais submetidos apenas a infusão de noradrenalina e grupo 2, constando de 15 animais também submetidos a infusão de noradrenalina e tiveram seus fígados retirados. Resultados Demonstram que, no grupo 2, antes da hepatectomia, as dosagens de glicemias arteriais foram maiores que as venosas, e após a retirada do órgão, estes valores se tornaram menores. Isto configurou inversão das curvas das glicemias. No grupo 1 não foi observada essa inversão. Conclusão - Conclui-se que esse comportamento das curvas de glicemia observadas nos animais do grupo 2 não se deve à ação da noradrenalina e significa liberação renal de glicose após a hepatectomia.

DESCRITORES - Hepatectomia. Glucose, metabolismo. Norepinefrina. Coelhos.

\footnotetext{
Trabalho realizado no Laboratório de Fígado e Transplantes da Disciplina de Moléstias do Aparelho Digestivo do Departamento de Cirurgia e Laboratório de Biologia Molecular da Faculdade de Ciências Médicas da Universidade Estadual de Campinas - UNICAMP, Campinas, SP.

Este trabalho contou com recursos financeiros da FAPESP - Fundação de Amparo à Pesquisa do Estado de São Paulo e está de acordo com a Declaração de Helsinque e as Normas Internacionais de Proteção aos Animais. Aprovado pelo Comitê de Ética Animal do Instituto de Biologia - UNICAMP.

* Mestre em Cirurgia pelo Departamento de Cirurgia da Faculdade de Ciências Médicas da FCM-UNICAMP. Professor Auxiliar do Departamento de Cirurgia da Faculdade de Medicina de Jundiaí, SP. Ex-Médico Assistente do Serviço de Cirurgia Geral e Digestiva II, Hospital Clínic i Provincial de Barcelona, Espanha. Former Honorary Registrar, Liver Unit, Queen Elizabeth Hospital, Birmingham, Inglaterra.

** Bióloga do Laboratório de Fígado do Núcleo de Medicina e Cirurgia Experimental da FCM-UNICAMP. Mestre em Biociências

*** Professor Titular e Chefe da Disciplina de Moléstias do Aparelho Digestivo, FCM-UNICAMP.

**** Professor Livre-Docente do Departamento de Clínica Médica e Chefe do Laboratório de Biologia Molecular, Departamento de Clínica Médica, FCM-UNICAMP.

Endereço para correspondências: Dr. Antonio Roberto Franchi-Teixeira - R. Quirino Amaral Campos, 115/11 - Cambuí - 13024-460 - Campinas, SP.
} 


\section{INTRODUÇÃO}

O transplante ortotópico de fígado (TOF) já constitui um tratamento efetivo para as doenças hepáticas terminais ${ }^{(4)}$.

Durante o transplante a retirada do órgão nativo leva a graves alterações hemodinâmicas, hematológicas e metabólicas no indivíduo ${ }^{(6,}$ 23). Dado que o fígado é peça fundamental no metabolismo da glicose, sua ausência por período definido acarreta grandes desarranjos na manutenção do estado metabólico normal ${ }^{(3,9,17)}$.

A fase anepática do transplante compreende o período entre a retirada do órgão nativo e o momento de reperfusão sangüínea do novo fígado, e neste período ocorrem alterações da regulação de glicose plasmática ${ }^{(17)}$. Durante essa fase, a hipoglicemia seria previsível devido ao fato de que a utilização periférica de glicose continua ocorrendo, enquanto o suprimento hepático está ausente. No entanto, na grande maioria dos doentes, esta hipoglicemia não ocorre. Pelo contrário, é observado um estado normo e até hiperglicêmico durante essa fase.

Também há relato de indução de coma hiperosmolar devido a infusão de grande quantidade de glicose na fase anepática ${ }^{(1)}$. Questionase, atualmente, qual seria a principal fonte de glicose na ausência do fígado, capaz de produzir energia necessária aos tecidos que somente dela se utilizam por períodos de tempo operatório que chegam a durar até duas horas.

Estudos realizados em grandes séries de transplantes demonstraram que doentes submetidos ao TOF (sem infusão alguma de glicose) não desenvolveram hipoglicemia durante o período de ausência do fígado ${ }^{(3)}$. Foi então advogado o fato de que a manutenção deste estado euglicêmico fosse decorrência da carga de glicose contida em produtos derivados do sangue (plasma humano, albumina e concentrado de hemácias), infundidos em larga escala durante o ato operatório $^{(3)}$. No entanto, a pequena quantidade de glicose presente nesses derivados não parece ser suficiente para manter estados de normoglicemia por períodos tão extensos. A fonte principal de glicose necessária para a manutenção da homeostase e consumo cerebral durante a ausência do fígado deve, portanto, ser de origem endógena e não exógena.

O papel do rim no metabolismo da glicose foi considerado de menor importância sob muitas circunstâncias ${ }^{(1)}$. No entanto, há indícios de que essa importância esteja sendo sub-valorizada ${ }^{(5,7,8,18,21,22)}$.

Evidências in vitro indicam que o rim é capaz de produzir e utilizar glicose ${ }^{(11,14)}$. CERSOSIMO et al. ${ }^{(7)}$, estudando cães conscientes com o uso de traçadores radioativos de carbono, demonstraram que existem, simultaneamente, produção e consumo intrarrenal de glicose. Aproximadamente $30 \%$ da glicose produzida e consumida no organismo desses cães se devem aos rins.

Recente trabalho na literatura estudou, pela primeira vez, a origem da produção de glicose durante as diversas fases do TOF, através do oferecimento de glicose marcada com deutério radioativo durante o TOF. Este trabalho demonstrou que havia produção endógena de glicose, sugerindo ser o rim o provável local de origem ${ }^{(13)}$.

Foi, então, desenvolvido um modelo animal com o objetivo de estudar as alterações dos níveis de glicemia, procurando determinar através das dosagens arteriais e venosas se o rim é possível local de produção de glicose. A criação deste modelo tem por objetivo mimetizar as condições fisiológicas da fase anepática do TOF, tornando possível estudar a influência do rim no oferecimento de glicose na presença e na ausência do fígado.

\section{MATERIAL E MÉTODOS}

Foram utilizados 20 coelhos domésticos (Oryctolagus cuniculus) variedade Nova Zelândia, do sexo masculino. Os animais foram divididos em dois grupos:

Grupo 1 - Constando de cinco animais que receberam infusão contínua de noradrenalina (NAdr) e tiveram seus fígados intactos durante todo procedimento

Grupo 2 - Constando de 15 animais que também receberam NAdr mas foram submetidos a hepatectomia total funcional.

A indução anestésica deu-se por meio de injeção intramuscular de $50 \mathrm{mg}$ de Zoletil $50^{\circledR}$ (cloridrato de tiletamina e zolazepan - Virbac Laboratórios, marca de Laboratoire Virbac, França). Os animais receberam $50 \mathrm{mg}$ de Zoletil $50^{\circledR}$ (tiletamina - zolazepan) por via endovenosa a cada 40 minutos e submetidos a traqueostomia cirúrgica para ventilação mecânica com oxigênio-ciclador Takaoka ${ }^{\circledR}$.

Os animais apenas receberam solução salina $(\mathrm{NaCl} 0,9 \%)$ e $\mathrm{NAdr}$ por bomba de infusão na taxa de $0,03 \mu \mathrm{g} / \mathrm{kg} / \mathrm{min}$ em veia periférica da orelha.

Procedeu-se à laparotomia e foi cateterizada a aorta em sua porção infra-renal. Foi acoplado a este cateter um sistema de aferição da pressão arterial média (PAM). A veia cava inferior foi cateterizada com sonda do tipo "venocath" de maneira que sua extremidade se situasse no interior da veia renal direita.

\section{Intervenção realizada nos animais do grupo 1}

Estes animais, já anestesiados e recebendo NAdr na taxa de infusão de $0,03 \mu \mathrm{g} / \mathrm{mL} / \mathrm{min}$, foram submetidos a uma primeira coleta sangüínea dos cateteres localizados na aorta e no interior da veia renal direita para dosagem de glicemia. Cinco minutos depois, ocorreu nova coleta e após mais 5 minutos, nova coleta. Após essa terceira coleta, a taxa de infusão de NAdr foi elevada para $0,10 \mu \mathrm{g} / \mathrm{mL} / \mathrm{min}$ e este momento foi designado como "tempo zero". Cinco minutos mais tarde foram colhidas novas amostras. Assim, procedeu-se a colheitas de novas amostras $10,15,25,35,45$, e 55 minutos após o tempo zero. 


\section{Intervenção realizada nos animais do grupo 2}

Os animais do grupo 2 foram anestesiados da mesma maneira e receberam a mesma droga vasoativa (NAdr) em doses iguais às dos animais do grupo 1. As coletas de sangue da aorta e veia renal direita para dosagem de glicemia foram realizadas também de maneira idêntica ao grupo 1 porém, no tempo definido como zero, foram submetidos a hepatectomia total funcional.

Para a realização da hepatectomia total funcional foi isolado o ligamento hepatoduodenal (artéria hepática e veia porta) com fio de algodão através do forame de de Winslow, realizando-se a manobra de Pringle. A isquemia arterial e venosa do parênquima hepático foi avaliada em todos os animais através de uma incisão feita a tesoura em cada um dos lobos hepáticos, de onde não ocorreu sangramento algum.

Cada amostra (arterial e venosa) foi apreciada quanto a seu conteúdo de glicose no Laboratório de Patologia Clínica do Hospital das Clínicas da UNICAMP. As dosagens de glicose foram expressas em mg/dL.

\section{Método Estatístico}

Foram utilizados métodos estatísticos não-paramétricos. O nível de rejeição para a hipótese de nulidade foi fixado sempre em valor igual ou menor que 0,05 .

Para avaliar as glicemias da aorta e da veia renal direita em ambos os grupos em relação às possíveis diferenças entre os tempos, foi usado o teste não-paramétrico para "k" amostras não-independentes de Friedman ${ }^{(19)}$.

Para avaliar as possíveis diferenças quanto a glicemia arterial e venosa em ambos os grupos em cada tempo específico, foi usado o teste nãoparamétrico para duas amostras não-independentes de Wilcoxon ${ }^{(19)}$.

\section{RESULTADOS}

O Gráfico 1 representa visualmente o que foi observado nos animais do grupo 1. A curva tracejada representa o comportamento da glicemia arterial ao longo do tempo. A curva contínua representa as glicemias venosas e o tempo "zero" marca o momento do aumento da taxa de infusão de NAdr. Os comportamentos das curvas de glicemia arterial e venosa deste Gráfico foram estudados estatisticamente pelo teste de Friedman, que analisa este comportamento ao longo do tempo.

A Tabela 1 traz os cálculos do $\chi 2$ calculado pelo teste de Friedman no Gráfico 1. O $\chi 2$ da curva de glicemia arterial resulta no valor 9,808 e quando aplicado à curva de glicemia venosa, resulta em 7,865. Ambos os valores não são estatisticamente significativos. Isto demonstra que a suposta elevação dos valores de glicemia arterial e venosa nos animais deste grupo (observada nas curvas do Gráfico 1) não é estatisticamente real, representando apenas uma tendência.

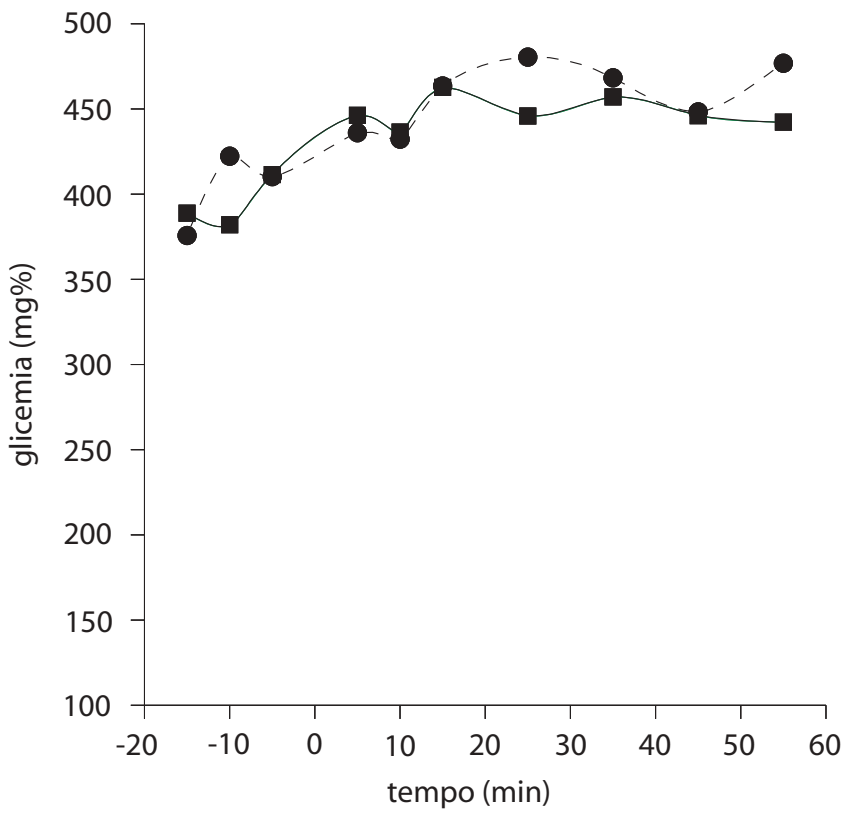

Obs.1: O tempo zero equivale ao momento da hepatectomia e da elevação da taxa de infusão de NAdr

Obs.2: Círculos e linha tracejada: curva de glicemias arteriais (aorta). Quadrados e linha contínua: curva de glicemias venosas (veia renal direita)

GRÁFICO 1 - Curvas das glicemias arteriais e venosas dos animais que receberam NAdr e não foram submetidos a hepatectomia total funcional (grupo 1)

TABELA 1 - Qui-quadrado calculado $(\chi 2$ calc) dos valores das glicemias arteriais e venosas dos animais do grupo 1 , calculados pelo teste de Friedman

\begin{tabular}{lcc}
\hline Grupo 1 & $\boldsymbol{\chi 2}^{\mathbf{c} \text { calc }}$ & Significância \\
\hline glicemia arterial & 9,808 & NS \\
glicemia venosa & 7,865 & NS \\
\hline
\end{tabular}

Os valores das glicemias arteriais e venosas do Gráfico 1 também foram comparados entre si em cada tempo específico pelo teste de Wilcoxon, como demonstrado na Tabela 2. Em apenas três tempos, a diferença calculada (z. calc) entre os valores de glicemia arterial e venosa foi significativa. Por conseguinte, em 7 tempos, a diferença entre os valores de glicemia arterial e venosa não existiu. Isto demonstra que não é possível identificar um comportamento definido das curvas de glicemia arterial e venosa.

O Gráfico 2 refere-se ao estudo das curvas de glicemias arteriais e venosas dos animais hepatectomizados (grupo 2). A curva tracejada representa a glicemia arterial (aorta) e a curva contínua, por sua vez, representa o comportamento das glicemias venosas ao longo do tempo. As quedas dos valores glicêmicos são observadas nitidamente neste gráfico. É possível notar, também, que a queda dos valores de glicemia venosa não é tão acentuada. Os valores das glicemias arteriais nos tempos 
negativos (coletas que antecederam a hepatectomia) são maiores que os venosos colhidos nos mesmos tempos. Entretanto, após a hepatectomia (tempos positivos), os valores das glicemias venosas decaem menos que das arteriais, configurando uma inversão das curvas.

TABELA 2 - Diferenças calculadas (z. calc) entre os valores de glicemias arteriais e venosas em cada tempo nos animais do grupo 1, calculadas pelo teste de Wilcoxon

\begin{tabular}{lcc}
\hline Tempos & z. calc & Significância \\
\hline-15 & $-0,674$ & NS \\
-10 & $-2,023^{*}$ & $\operatorname{sim}$ \\
-5 & $-0,135$ & NS \\
5 & $-0,405$ & NS \\
10 & $-0,405$ & NS \\
15 & $-0,405$ & NS \\
25 & $2,023^{*}$ & sim \\
35 & $-0,944$ & NS. \\
45 & $-0,135$ & NS \\
55 & $-2,023^{*}$ & sim \\
\hline
\end{tabular}

A Tabela 3 traz os valores dos $\chi 2$ das curvas de glicemia arterial e venosa calculados pelo teste de Friedman. Para as glicemias arteriais o resultado foi de 114,529, valor estatisticamente significativo. Quando aplicado para curva das glicemias venosas, o $\chi 2$ calculado resulta no valor de 83,948, número também estatisticamente significativo. Ambos os resultados demonstram que as quedas das glicemias na artéria e na veia dos animais deste grupo (visíveis no Gráfico 2) são estatisticamente reais e não significam apenas uma tendência.

Os valores das glicemias arteriais e venosas do Gráfico 2 também foram submetidos ao teste de Wilcoxon, como demonstrado na Tabela 4. Este teste compara os valores de glicemia arterial e venosa em cada tempo e calcula sua diferença, com o objetivo de averiguar se estes pontos realmente são diferentes entre si. Em todos os tempos estudados, com exceção do tempo $=5 \mathrm{~min}$, a diferença calculada (z. calc) foi estatisticamente significativa. $\mathrm{O}$ tempo $\mathrm{t}=5 \mathrm{~min}$ é próximo do ponto de inflexão das curvas, portanto é compreensível que a diferença entre o valor de glicemia arterial e venosa não seja estatisticamente significativa apenas neste tempo. Portanto, a inversão das curvas que se observa no Gráfico 2 é real.

TABELA 3 - Qui-quadrado calculado $(\chi 2$ calc) dos valores das glicemias arteriais e venosas dos animais do grupo 2, calculado pelo teste de Friedman

\begin{tabular}{lcc}
\hline Grupo 1 & $\boldsymbol{\chi 2}$ calc & Significância \\
\hline glicemia arterial & $114,529^{*}$ & $\operatorname{sim}$ \\
glicemia venosa & $83,948^{*}$ & $\operatorname{sim}$ \\
\hline
\end{tabular}

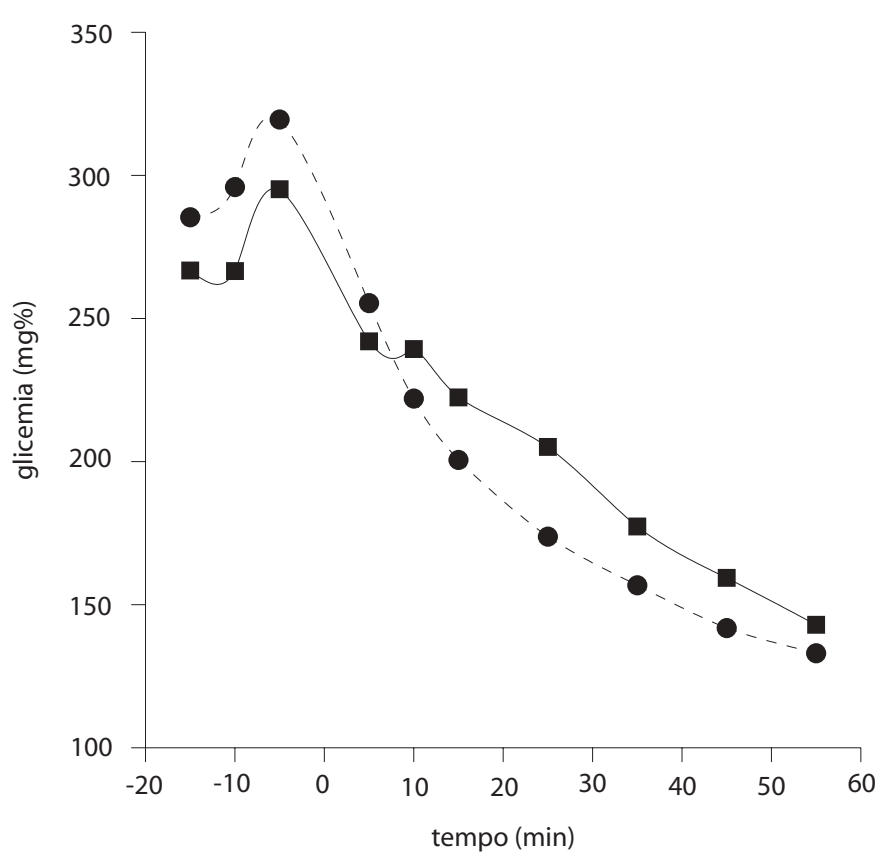

Obs.1: O tempo zero equivale ao momento da hepatectomia e da elevação da taxa de infusão de NAdr

Obs.2: Círculos e linha tracejada: curva de glicemias arterias (aorta)

Quadrados e linha contínua: curva de glicemias venosas (veia renal direita)

GRÁFICO 2 - Curvas das glicemias arteriais e venosas dos animais que receberam NAdr e foram submetidos a hepatectomia total funcional (grupo 2)

TABELA 4 - Diferenças calculadas (z. calc) entre os valores de glicemias arteriais e venosas em cada tempo nos animais do grupo 2, calculadas pelo teste de Wilcoxon

\begin{tabular}{lcc}
\hline Tempos & z. calc & Significância \\
\hline-15 & $-3,108^{*}$ & $\operatorname{sim}$ \\
-10 & $-2,982^{*}$ & $\operatorname{sim}$ \\
-5 & $-2,161^{*}$ & $\operatorname{sim}$ \\
5 & $-1,423$ & $\mathrm{NS}$ \\
10 & $-2,386^{*}$ & $\operatorname{sim}$ \\
15 & $-3,268^{*}$ & $\operatorname{sim}$ \\
25 & $-3,480^{*}$ & $\operatorname{sim}$ \\
35 & $-3,238^{*}$ & $\operatorname{sim}$ \\
45 & $-3,26^{*}$ & $\operatorname{sim}$ \\
55 & $-3,239^{*}$ & $\operatorname{sim}$ \\
\hline
\end{tabular}

\section{DISCUSSÃO}

Nos dois grupos de animais observou-se hiperglicemia acentuada nas dosagens arteriais e venosas desde o início do experimento. Isto 
pode ser explicado porque os animais vinham sendo alimentados por toda a noite anterior, com ração balanceada ad libitum. Isto foi uma estratégia proposital porque o objetivo era estudar a capacidade de reserva energética renal após a hepatectomia. Outro fato importante é que coelhos são animais que se estressam facilmente e podem apresentar liberação de catecolaminas endógenas que podem ter efeito hiperglicemiante sistêmico ${ }^{(20)}$.

\section{Resultados dos animais do grupo 1}

As curvas de glicemia arterial e venosa dos animais do grupo que não foi submetido a hepatectomia (grupo 1) não diminuem ao longo do tempo, pelo contrário, tendem a subir ligeiramente. Isto pode ser explicado pelo fato de que este grupo de animais permanece com o fígado sob efeito hiperglicemiante da NAdr. Esta tem ação no metabolismo de carboidratos porque estimula os receptores $\beta$ em fígado e pâncreas, reduz a secreção de insulina e eleva a glicemia sérica através da mobilização das reservas energéticas hepáticas ${ }^{(12,20)}$. Os testes estatísticos aplicados ao gráfico das glicemias destes animais (Gráfico 1), demonstraram dois fatos importantes: primeiramente, as curvas de glicemia arterial e venosa não apresentam comportamento definido ao longo do tempo. A tendência à subida, detectada visualmente no gráfico, não é estatisticamente significativa (teste de Friedmann). Em segundo lugar, não existe diferença significativa entre as dosagens de glicemia arterial e venosa na maioria dos tempos (teste de Wilcoxon). Não existe, portanto, nenhum padrão de comportamento estatístico definido de inversão das curvas de glicemia arterial e venosa ao longo do experimento nos animais desse grupo.

\section{Resultados dos animais do grupo 2}

Os animais do grupo 2 também apresentaram hiperglicemia acentuada já no início do procedimento, tanto nas dosagens arteriais, quanto nas venosas, como os animais do grupo anterior. Isto pode ser explicado pelas razões já discutidas. No entanto, observa-se acentuada queda nas glicemias arteriais e venosas após a realização da hepatectomia total funcional. O estudo do comportamento das curvas de glicemia arterial e venosa ao longo do tempo (pelo teste de Friedman) revela que existe uma queda estatisticamente real. Isto demonstra que o fígado é fundamental na manutenção da glicemia sérica no coelho e mesmo sob ação hiperglicemiante da NAdr, a hipoglicemia não pôde ser evitada neste grupo submetido a hepatectomia. O Gráfico 2 também revela que antes da hepatectomia, a curva das glicemias arteriais (tracejada) mantém-se nitidamente acima da curva das glicemias venosas (contínua). Após a hepatectomia, observa-se uma inversão no padrão das curvas, estudada pelo teste de Wilcoxon, que compara as curvas ponto-a-ponto, e foi significativa. Este é considerado o ponto-chave deste estudo, pois deste fato decorre a hipótese de que o rim passa a liberar glicose após a retirada do fígado.

Carnívoros e aves de rapina possuem maior capacidade neoglicogênica que os herbívoros, talvez pelo fato de que aqueles são obrigados a passar por períodos longos de jejum, enquanto estes têm alimento disponível na natureza. Aves carnívoras, como o urubu, têm maior capacidade de se manterem euglicêmicas após jejum prolongado que as aves graníferas, como o pombo ${ }^{(10,15)}$. O mesmo acontece com os mamíferos carnívoros. Isto poderia ser explicado por diferenças de hábitos alimentares, por variação entre as espécies, ou pela distribuição das enzimas neoglicogênicas nos diversos compartimentos intracelulares ${ }^{(15)}$. Por isso, é possível que a capacidade enzimática glicogenética renal nos coelhos (animal exclusivamente herbívoro) não seja extremamente desenvolvida. Isto pode explicar o fato de que, apesar da curva de glicemia venosa haver sobrepujado a curva de glicemia arterial após a hepatectomia, esta liberação renal de glicose não foi suficiente para evitar hipoglicemia sistêmica nos animais hepatectomizados (grupo 2).

Uma consideração importante é o fato de que foi cateterizada apenas a veia renal direita dos animais para dosagem de glicemia liberada pelo rim direito. Como os estímulos de neoglicogênese renal são sistêmicos, conclui-se que o rim esquerdo também liberou glicose na mesma taxa que o rim direito. No entanto, esta glicose liberada pelo rim esquerdo diluiu-se na circulação sistêmica de retorno ao coração e contribuiu para elevar a glicose dosada na aorta. É, pois, dedutível que a diferença entre as glicoses venosas e arteriais dosadas após a hepatectomia seja maior que aquela aferida neste experimento. Para medir tal diferença real, seria necessário realizar uma nefrectomia esquerda antes da hepatectomia total funcional, a fim de evitar esta interferência nas dosagens de glicose arterial.

Em resumo, os resultados deste estudo demonstram que após a hepatectomia total funcional em coelhos anestesiados, há queda acentuada nos níveis glicêmicos da aorta e da veia renal. Ocorrem alterações metabólicas que resultam em liberação de glicose na veia renal, porém esta é insuficiente para manter os níveis de glicemia. Isto vem ao encontro de recentes trabalhos publicados na literatura que demonstram que o papel do rim no metabolismo energético-glicêmico está sendo subestimado ${ }^{(2,7,13)}$. Ao contrário do que atualmente é descrito no livros-texto, é provável que o rim desempenhe papel-chave no metabolismo glicêmico, principalmente nas situações onde o fígado está incapacitado ou ausente.

O melhor conhecimento sobre a capacidade energética renal pode vir a contribuir para o entendimento do binômio rim-fígado nos hepatopatas crônicos. Há indícios clínicos sugerindo que doentes cirróticos dialíticos precisam de melhor controle glicêmico durante a fase anepática do transplante de fígado, necessitando, eventualmente, infusão suplementar dessa hexose. 
Franchi-Teixeira AR, Jorge GL, Leonardi LS, Saad MJA. Estudo da liberação renal de glicose em coelhos submetidos a hepatectomia total funcional e infusão de noradrenalina

Franchi-Teixeira AR, Jorge GL, Leonardi LS, Saad MJA. Renal glucose release in rabbits submitted to total functional hepatectomy and norepinephrine infusion. Arq Gastroenterol 2001;38(3):183-188.

ABSTRACT -Aim - To study the possible endogenous sources of glucose in the absence of the liver (equivalent to the anhepatic period of liver transplantation). Material and Methods - A experimental model of total functional hepatectomy in anesthetised rabbits was developed. The aorta and the right renal vein were catheterised in order to collect blood samples to measure glucose contents. The animals were divided into two groups: group 1, 5 animals underwent only norepinephrine infusion; group 2, 15 animals underwent norepinephrine infusion and submitted to total functional hepatectomy. Results - In group 2, before the hepatectomy, arterial glucose levels were higher than venous ones and after the liver removal, the venous levels became higher than the arterial ones. This pattern showed an inversion in the glicemic curves. In group 1 this pattern was not observed. Conclusion - The glicemic curves behavior observed in group 2 is not due to norepinephrine infusion, but represents renal glucose release after total functional hepatectomy.

HEADINGS - Hepatectomy. Glucose, metabolism. Norepinephrine. Rabbits.

\section{REFERÊNCIAS BIBLIOGRÁFICAS}

1. Aber GM, Morris LO, Housley E. Gluconeogenesis by the human kidney. Nature 1966;212:1589-90

2. Arem R. Hypoglycemia associated with renal failure. Endocrin Metab Clin North Am 1989;18:103-21.

3. Atchison SR, Rettke SR, Fromme GA, Janossy TA, Kunkel SE, Williamson KR, Perkins JA, Rakela J. Plasma glucose concentrations during liver transplantation. Mayo Clin Proc 1989;64:241-5.

4. Bismuth $\mathrm{H}$. The need for a consensus agreement of indications of liver transplantation. Hepatology 1994;20(supl 1):1S-2S

5. Bergman H, Drury DR. The relationship of kidney function to the glucose utilization of the extra abdominal tissues. Am J Physiol 1938;124:279-84.

6. Carmichael FL, Lindop MJ, Farman JV. Anesthesia for hepatic transplantation: cardiovascular and metabolic alterations and their management. Anesth Analg 1985;68:108-16.

7. Cersosimo E, Judd RL, Miles JM. Insulin regulation of renal glucose metabolism in conscious dogs. J Clin Invest 1994;93:2584-9.

8. Cherrington AD, Wasserman DH, McGuiness OP. Renal contribution to glucose production after a brief fast: fact or fancy. J Clin Invest 1994;93:2203.

9. DeWolf AM, Kang YG, Todo S, Kam I, Francavilla AJ, Polimeno L, Lynch S, Starzl TE. Glucose metabolism during liver transplantation in dogs. Anesth Analg 1987;66:76-80.

10. Foss MC. Metabolismo de carboidratos e lipídios e dieta hiperprotéica (livre de carboidrato) [tese de doutoramento]. Ribeirão Preto: Faculdade de Medicina de Ribeirão Preto - Universidade de São Paulo; 1978.

11. Guder G, Wagner S, Wirthensohn G. Metabolic fuels along the nephron: pathways and intracellular mechanism of interaction. Kidney Int 1989;29:41-5.

12. Harris RA. Carbohydrate metabolism. 1: Major metabolic pathways and there control. In: Devlin TM, editor. Textbook of biochemistry with clinical correlations. New York: Willey-Liss; 1997. p.267-333.
13. Joseph SE, Heaton N, Potter D, Pernet A, Umpleby MA, Amiel AS. Renal glucose production compensates for the liver during the anhepatic phase of liver transplantation. Diabetes 2000;49:450-6.

14. Krebs HA, Hems R, Gascoyne T. Renal gluconeogenesis iv - gluconeogenesis from substrate combinations. Acta Biol Med Germ 1963;11:607-15.

15. Linder C. Metabolismo de carboidratos e lipídios no urubu (coragyps atratus Bechts) [tese de Livre-Docência]. Botucatu: Faculdade de Ciências Médicas e Biológicas de Botucatu - Universidade Estadual Júlio de Mesquita Filho; 1971.

16. Machado MCC, Cunha JEM da, Margarido NF, Bachella T, Gonçalves L, Raia AA. Hyperosmolar coma associated with clinical liver transplantation. Int Surg 1976;61:368-9.

17. Palnaes-Hansen C, Boesby S, Kirkegaard P. Glucose homeostasis after ortotopic liver transplantation in the pig. Transplantation 1991;51:587-9.

18. Schoolwerth AC, Smith BC, Culpepper RM. Renal gluconeogenesis. Miner Electrolyte Metab 1988;14:347-61

19. Siegel S. Estatística não-paramétrica (para as ciências do comportamento). São Paulo: McGraw-Hill; 1975.

20. Stoelting RK. Sympathomimetics. In: Pharmacology and physiology in anesthetic practice. Philadelphia: J.B. Lippincott; 1987. p.251-68.

21. Stumvoll M, Chintalapudi U, Perrielo G, Welle S, Gutierrez O, Gerich J. Uptake and release of glucose by the human kidney. Postabsorptive rates and responses to epinephrine. J Clin Invest 1995;96:2528-33.

22. Stumvoll M. [The human kidney as an important producer of glucose]. Med Klin 1998;93:300-6.

23. Tullock WC, Pinsky MR, Shaw B, Martin DJ. Metabolic changes during the anhepatic phase of liver transplantation. Anesthesiology 1984;61:A271. 